\title{
RENDITE UND KAPITALKOSTEN
}

\author{
Jörg Laitenberger*
}

Aktuelle Version: 21. April 2004

\section{Überblick}

Zur Bewertung von Investitionsprojekten mit mehrperiodigen Zahlungen können die erwarteten Zahlungen mit geeigneten Kapitalkosten diskontiert werden. Die Kapitalkosten werden in der Regel als die erwartete einperiodige Rendite der Investition bestimmt, wobei häufig auf Kapitalmarktmodelle wie das CAPM zurückgegriffen wird, die die erwartete Rendite als die Summe der risikolosen Rendite und einer Risikoprämie ermitteln. In dieser Arbeit wird gezeigt, dass die einperiodigen Renditen nur unter bestimmten Annahmen die geeigneten Kapitalkosten darstellen. Wenn zwischen den einperiodigen Renditen Autokorrelation auftritt, dann ist ein zusätzlicher Korrekturterm bei der Ermittlung der Kapitalkosten zu berücksichtigen.

Im weiteren Verlauf der Arbeit wird analysiert, wie sich in Abhängigkeit der zeitlichen Aufösung der Unsicherheit die Kapitalkostenformeln ändern. Dabei stellt sich heraus, dass die Annahmen an die Risikoauflösung für die Zahlungen der Investition alleine keinerlei erklärende Kraft haben. Nur in Verbindung mit Annahmen an die Entwicklung der Zustandspreise können einfache und intuitive Relationen hergeleitet werden.

Keywords: Unternehmensbewertung, Kapitalkosten, Autokorrelierte Renditen

JEL-class.: J 31, G 46

${ }^{*}$ Lehrstuhl für Banken und Finanzierung der Universität Hannover, Königsworther Platz 1, 30167 Hannover, jl@wacc.de. Ich danke dem Verein zur Förderung der Zusammenarbeit zwischen Lehre und Praxis am Finanzplatz Hannover e.V. für seine Unterstützung. 


\section{RENDITE UND KAPITALKOSTEN}

\section{Einleitung}

Die moderne Finanzmarkttheorie auf arbitragefreien Märkten ermittelt den Wert eines Wertpapiers grundsätzlich als den Erwartungswert der als Zufallsvariablen aufgefassten zukünftigen Zahlungen der Wertpapiere multipliziert mit einem zustandsabhängigen Preisvektor. Bewertungsmodelle für derivative Finanzinstrumente (Optionen, Futures) und die Zinsstruktur haben in den letzten dreißig Jahren die Welt des Risikomanagements und den Wertpapierhandel revolutioniert. $\mathrm{Zu}$ ersteren gehören die Black-ScholesFormel sowie das Binomialmodell von Cox, Ross und Rubinstein. Zu letzterem gehören u.a. die Bewertungsmodelle von Vasicek, von Cox, Ingersoll und Ross oder das Heath-Jarrow-Morton-Modell. Im Vergleich dazu existieren zur Bewertung von Aktien und allgemeiner von Unternehmen kaum Ergebnisse, die das Verfahren der zustandsabhängigen Preisvektoren nutzen. Dies liegt in erster Linie daran, dass aufgrund der ungleich höheren Zahl an Erklärungsvariablen für Aktienkursentwicklungen die Bestimmung eines geeigneten stochastischen Preisfunktionals für Aktienbewertungen wesentlich schwieriger ist und bisher empirisch nicht durchgeführt worden ist. Solange aber keine Theorie zur Bestimmung der relevanten Zustandspreise existiert, muss jede Untersuchung zur Bewertung von Investitionen, die diese verwendet, eine akademische Leerformel bleiben.

Die Literatur zur Bewertung von Aktien und allgemeiner von Unternehmen geht daher einen anderen Weg. Hier wird in der Regel vorgeschrieben, die erwarteten Zahlungen mit einem risikoadjustierten Diskontierungszinssatz abzuzinsen. Der risikoadjustierte Diskontierungszinssatz soll dabei die Opportunitätskosten einer entgangenen Rendite bei einer Anlage in eine vergleichbare alternative Investition wiedergeben. Dabei wird in der Regel vorgeschlagen die erwarteten einperiodigen Renditen der Investition als Kapitalkosten heranzuziehen. Diese werden in praktischen Anwendungen in der Regel mit Hilfe des CAPM als die Summe aus risikolosem Zins und einer Risikoprämie berechnet. Es ist allerdings äußerst unwahrscheinlich, dass sowohl der risikolose Zins als auch die Risikoprämie über die Dauer der Investition konstant bleiben. Brennan (1997) hat daher vorgeschlagen, variable Kapitalkosten für unterschiedliche Perioden der Investitionsdauer zu verwenden. Brennan leitet anhand eine ökonometrischen Modells für die erwarteten Renditen eine Zinsstrukturkurve für die Diskontierungsfaktoren her. Jüngst haben Ang und Liu (2002) diesen Ansatz erweitert, indem sie 
auch Schwankungen des Betas der Investition modellieren.

Der vorliegende Beitrag untersucht unter welchen Bedingungen an die gemeinsame stochastische Entwicklung sowohl der Zahlungen des Bewertungsobjektes als auch des stochastischen Marktpreisfunktionals die Methode der Kapitalkosten mit einer arbitragefreien Wertbestimmung kompatibel ist. Dabei stellt sich heraus, dass bei mehrperiodigen Investitionsprojekten die einperiodigen Renditen der Investition nur unter sehr eingeschränkten Bedingungen als Kapitalkosten geeignet sind, nämlich nur dann, wenn die Renditen des Projektes stochastisch unkorreliert sind. Dies wiederum wird aber in der Regel nur dann erreicht, wenn die bedingten Kovarianzen des Unternehmenswertes mit dem Marktpreisfunktional zu allen zukünftigen Zeitpunkten deterministisch sind. Wenn dies nicht der Fall ist, dann muss bei mehrperiodigen Investitionsprojekten zur Bestimmung der Kapitalkosten ein Korrekturfaktor zur erwarteten einperiodigen Rendite hinzutreten. Empirische Evidenz für autokorrelierte Renditen haben zumindest für den US-amerikanischen Kapitalmarkt unter anderem Fama und French (1988), Lo und Mackinlay (1988) und Poterba und Summers (1988) vorgelegt.

Im weiteren Verlauf wird analysiert, wie sich in Abhängigkeit der zeitlichen Auflösung der Unsicherheit die Kapitalkostenformeln ändern. Auch der in der Literatur diskutierte Extremfall eines sich schlagartig auflösenden Risikos führt nur dann dazu, dass die risikolose Rendite als Kapitalkosten herangezogen werden kann, wenn die bedingte erwartete Rendite der Zahlungen eine sichere Größe ist. Ansonsten können nämlich die zeitlichen Bewertungen der Zahlungen auch in den Zwischenperioden zu unsicheren Renditen führen, die eine Bewertung mit dem risikolosen Zinssatz verbieten. Allgemein gilt, dass die Annahmen an die Risikoauflösung für die Zahlungen der Investition alleine keinerlei erklärende Kraft haben. Nur in Verbindung mit Annahmen an die Entwicklung der Zustandspreise können einfache und intuitive Relationen hergestellt werden.

Der Aufsatz ist wie folgt aufgebaut. Der folgende Abschnitt stellt verschieden Bewertungsverfahren auf arbitragefreien Märkten vor. Während die Abschnitte 2.1 und $2.3 \mathrm{im}$ Wesentlichen bekannte Relationen aus der Finanzmarkttheorie rekapitulieren, wird in Abschnitt 2.2 die Anwendung der Risikozuschlagstheorie untersucht und eine konsistente Definition für die Kapitalkosten geliefert. Im darauf folgenden Abschnitt werden Rendite und Kapitalkosten für in der Literatur diskutierte Formen der Risikoauflösung untersucht. 


\section{Bewertungsgleichungen}

Dieser Aufsatz handelt von Unternehmenswerten im Rahmen der Kapitalmarkttheorie auf arbitragefreien Märkten. Das bedeutet, dass die Annahmen an einen reibungslosen Marktverkehr sowohl für die am Markt gehandelten Wertpapiere als auch für das zu bewertende Unternehmen gelten. Es werden demnach die folgenden Annahmen getroffen:

- Alle Wertpapiere und die Anteile an dem zu bewertenden Unternehmen sind zu jedem Zeitpunkt und in beliebiger Stückzahl handelbar. Insbesondere sind Leerverkäufe unbegrenzten Ausmaßes erlaubt und die Anteile sind beliebig teilbar.

- Es fallen keinerlei Transaktionskosten an. Kauf- und Verkaufspreise sind immer gleich.

- Die Zusammensetzung der Anteilseigner eines Unternehmens hat keinerlei Auswirkungen auf dessen zukünftige Erlöse.

- Es werden zu keinem Zeitpunkt Steuern gezahlt.

Mit diesen Annahmen wird ein Rahmen gesetzt, in dem sich die folgende Diskussion bewegen wird. Es wird damit nicht ausgeschlossen, dass viele Bewertungsverfahren in der Realität unter vollkommen anderen Bedingungen stattfinden. Es ist zum Beispiel allgemein bekannt und auch empirisch nachgewiesen, dass die Eigentümerstruktur eines Unternehmens durch Effekte moralischen Risikos einen nennenswerten Einfluss auf den Unternehmenswert haben kann. Dies dürfte insbesondere bei der in Deutschland vorherrschenden mittelständisch geprägten Unternehmerstrutur bei Bewertungsverfahren, in denen Eigentümer involviert sind, die Alleininhaber ihrer Unternehmen sind oder im Rahmen von Familienunternehmen wesentliche Anteile halten, durchaus von Bedeutung sein. Für diese Fälle sind andere Bewertungsverfahren heranzuziehen.

\subsection{Zustandspreise}

Die Kapitalmarkttheorie auf arbitragefreien Märkten erlaubt die Bewertung von Zahlungsströmen mit Hilfe von so genannten Zustandspreisen, die die Preise der Wertpapiere in einem linearen Verhältnis ihrer zukünftigen Zahlungen setzt. Der Rahmen für die Bewertung mit Zustandspreisen ist allgemeiner Lehrbuchstof ${ }^{1}$ soll deshalb hier nur sehr kurz abgehandelt werden.

\footnotetext{
${ }^{1}$ Man schlage zum Beispiel bei Wilhelm (1985) nach.
} 
Man betrachte einen Kapitalmarkt mit Unsicherheit, die durch einen Wahrscheinlichkeitsraum $(\Omega, \mathcal{F}, P)$ dargestellt wird. $s \in \Omega$ bezeichne einen primitiven Zustand der Ökonomie. $\mathcal{F}_{t}$ sei die Partition der Ereignisse, die zum Zeitpunkt $t$ bekannt sind und die Folge der Partitionen $\mathcal{F}_{t=0, \ldots, T}$ sei eine Filtration des Zustandsraums ${ }^{2}$ Es werden diskrete Zeitperioden $t=0, \ldots, T$ betrachtet, wobei $T=\infty$ möglich ist. Ein Wertpapier liefert einen unsicheren Zahlungsstrom von zukünftigen Dividenden $d_{t}$. Der Preis des Wertpapiers in $t$ sei $V_{t}$. Sowohl $d_{t}$ und $V_{t}$ sind $\mathcal{F}_{t}$-messbare Zufallsvariablen. Die Annahme arbitragefreier Märkte garantiert die Existenz eines strikt positiven, messbaren Prozess $\pi_{t}$, so dass der Preis eines Wertpapiers sich wie folgt aus seinen Auszahlungen in der folgenden Periode ergibt:

$$
V_{t}=\mathrm{E}_{t}\left[\frac{\pi_{t+1}}{\pi_{t}}\left(d_{t+1}+V_{t+1}\right)\right],
$$

wobei $\mathrm{E}_{t}[\cdot]$ den bedingten Erwartungswert zum Zeitpunkt $t$ bezeichnet. $\pi_{t}$ ist der Zustandspreis oder der stochastische Diskontierungsfaktor.

Für ein Wertpapier mit mehrperiodigen Zahlungen führt die wiederholte Anwendung von Gleichung (1) zusammen mit harmlosen technischen Annahmen (der Prozess $\mathrm{E}_{t}\left[\pi_{\tau} d_{\tau}\right]$ sollte gegen Null konvergieren, wenn $\tau \rightarrow \infty$ ) zu einer Formel für den Wertpapierpreis als Summe aller zukünftigen abdiskontierten Dividenden:

$$
V_{t}=\sum_{\tau=t+1}^{T} \mathrm{E}_{t}\left[\frac{\pi_{\tau}}{\pi_{t}} d_{\tau}\right] .
$$

Der Preis eines einperiodigen risikolosen Wertpapiers, also eines Wertpapiers, das in der Periode $t+1$ eine sichere Zahlung in Höhe von eins und in allen anderen Perioden von Null liefert, ist gleich

$$
\mathrm{E}_{t}\left[\frac{\pi_{t+1}}{\pi_{t}}\right]
$$

Die Rendite einer sicheren Investition von $t$ nach $t+1$ sei der risikolose Zins $i_{t+1}$ :

$$
1+i_{t+1}=\frac{1}{\mathrm{E}_{t}\left[\frac{\pi_{t+1}}{\pi_{t}}\right]}
$$

Die Rendite einer risikolosen Investition von $t$ bis $t+\tau$ sei:

$$
1+i_{t, \tau}=\frac{1}{\mathrm{E}_{t}\left[\frac{\pi_{\tau}}{\pi_{t}}\right]}
$$

\footnotetext{
${ }^{2}$ Für weitere Details konsultiere man Standardlehrbücher der Wahrscheinlichkeitstheorie, z.B. Bamberg und Baur (2001).
} 
Wird als Diskontierungsfaktor der riskolose Zins verwendet, wird aus dem Zustandspreis $\pi_{t}$ ein Bewertungsindex:

$$
q_{t, \tau}=\frac{\frac{\pi_{\tau}}{\pi_{t}}}{\mathrm{E}_{t}\left[\frac{\pi_{\tau}}{\pi_{t}}\right]}
$$

und die Bewertungsformeln (1) und (2) ergeben sich zu

$$
V_{t}=\frac{\mathrm{E}_{t}\left[q_{t, t+1}\left(d_{t+1}+V_{t+1}\right)\right]}{1+i_{t, t+1}}
$$

und

$$
V_{t}=\sum_{\tau=t+1}^{T} \frac{\mathrm{E}_{t}\left[q_{t, \tau} d_{\tau}\right]}{1+i_{t, \tau}} .
$$

Mit der Kovarianzidentität lassen sich diese Gleichungen auch wie folgt schreiben

$$
V_{t}=\frac{\mathrm{E}_{t}\left[d_{t+1}+V_{t+1}\right]+\operatorname{Cov}_{t}\left[d_{t+1}+V_{t+1}, q_{t, t+1}\right]}{1+i_{t, t+1}},
$$

bzw.

$$
V_{t}=\sum_{\tau=t+1}^{T} \frac{\mathrm{E}_{t}\left[d_{\tau}\right]+\operatorname{Cov}_{t}\left[d_{\tau}, q_{t, \tau}\right]}{1+i_{t, \tau}}
$$

da $\mathrm{E}_{t}\left[q_{t, t+\tau}\right]=1$.

Auf einem arbitragefreien Finanzmarkt kann also jedes Wertpapier und demnach auch jedes Unternehmen bewertet werden, wenn ausreichend Kenntnisse über die stochastischen Verteilungen der zukünftigen Dividenden als auch der stochastischen Diskontierungsfaktoren vorhanden sind. Gleichung (7) zeigt, dass nicht die vollständige Verteilungen sondern nur deren Erwartungswerte sowie die Kovarianzen mit dem Bewertungsindex und die Zinsstrukturkurve bekannt sein müssen, was allerdings immer noch eine ziemliche Menge an Informationen ist.

\subsection{Die Risikozuschlagsmethode}

Die traditionelle Unternehmensbewertunsliteratur kennt zwei Methoden der Unternehmensbewertung: die Sicherheitsäquivalentmethode und die Risikozuschlagsmethode. Erstere soll hier nicht weiter betrachtet werden. Die Risikozuschlagsmethode, die auch die in der Praxis meist verwendete Methode ist, sieht vor, dass die erwarteten zukünftigen Zahlungen mit einem 
adäquaten risikoangepassten Diskontierungszinssatz abgezinst werden. Der Diskontierungszinssatz wird dabei als die Kapitalkosten der Investition verstanden und entspricht der Rendite einer vergleichbaren Investition am Kapitalmarkt. Die zu bewertende Investition übernimmt damit automatisch die selbe Rendite an wie die herangezogenen Alternativanlage. Damit erhalten zumindest die Kapitalkosten einer einperiodigen Investition eine einfache ökonomische Interpretation als erwartete Rendite der Investition. Die Rendite von Periode $t-1$ zu $t$ ist:

$$
r_{t}:=\frac{V_{t}+d_{t}}{V_{t-1}}-1
$$

Wenn die zukünftigen Erträge unsicher sind, wird auch $r_{t}$ eine unsichere Größe sein. Da

$$
V_{t-1}\left(1+r_{t}\right)=d_{t}+V_{t}
$$

ergibt sich

$$
V_{t-1}=\frac{\mathrm{E}_{t-1}\left[d_{t}+V_{t}\right]}{1+\mathrm{E}_{t-1}\left[r_{t}\right]} .
$$

Also ist $\frac{1}{1+\mathrm{E}_{t-1}\left[r_{t}\right]}$ der Diskontierungsfaktor einer einperiodischen Investition. Dies ist auch das gängige Verfahren, nach dem sowohl in der Praxis als auch bei empirischen Untersuchungen zu Aktienrenditen die Kapitalkosten bestimmt werden.

Bei Investitionsprojekten mit Auszahlungen in mehreren zukünftigen Perioden kann dieses Ergebnis auf zweierlei Arten verallgemeinert werden. Einmal ist es möglich die Zahlungen in jeder einzelnen Periode mit den ihren eigenen Kapitalkosten zu bewerten 3 Die Bestimmung dieser Kapitalkosten ist aber nahezu unmöglich, da zur Bestimmung der Rendite einer einzelnen Zahlung deren Wertbeitrag zum Gesamtunternehmenswert bekannt sein müsste. Empirisch zu messen ist aber nur die Rendite der gesamten Investition, die offensichtlich ein gewichteter Durchschnitt der Renditen aller zukünftigen Cashflows ist 4 Alternativ werden die einperiodigen Renditen der gesamten Investition wiederholt angewendet. Dies führt allerdings nur dann zu einem korrekten Unternehmenswert, wenn die einperiodigen Renditen untereinander unkorreliert sind. Sonst müssen gewissen Korrekturterme in den Kapitalkosten berücksichtigt werden. Da

$$
V_{t-1}\left(1+\mathrm{E}_{t-1}\left[r_{t}\right]\right)=\mathrm{E}_{t-1}\left[d_{t}+V_{t}\right]
$$

\footnotetext{
${ }^{3}$ So zum Beispiel bei Brennan (1997) und Ang und Liu (2002).

${ }^{4}$ Vergleiche Rapp (2003).
} 
hat man bei einer Investition über zwei Perioden

$$
\mathrm{E}_{t-2}\left[V_{t-1}\right]=\frac{\mathrm{E}_{t-2}\left[d_{t}+V_{t}\right]}{1+\mathrm{E}_{t-2}\left[r_{t}\right]+\frac{\operatorname{Cov}_{t-2}\left[V_{t-1}, \mathrm{E}_{t-1}\left[r_{t}\right]\right]}{\mathrm{E}_{t-2}\left[V_{t-1}\right]}}
$$

und

$$
V_{t-2}=\frac{\mathrm{E}_{t-2}\left[d_{t-1}\right]}{1+\mathrm{E}_{t-2}\left[r_{t-1}\right]}+\frac{\mathrm{E}_{t-2}\left[d_{t}+V_{t}\right]}{\left(1+\mathrm{E}_{t-2}\left[r_{t-1}\right]\right)\left(1+\mathrm{E}_{t-2}\left[r_{t}\right]+\frac{\operatorname{Cov} t-2_{t-1}\left[V_{t-1}, \mathrm{E}_{t-1}\left[r_{t}\right]\right]}{\mathrm{E}_{t-2}\left[V_{t-1}\right]}\right)} .
$$

Daher ist $\frac{1}{1+\mathrm{E}_{t-2}\left[r_{t}\right]}$ nur dann der geeignete Diskontierungsfaktor zur Bewertung der Erträge in der zweiten Periode, wenn

$$
\operatorname{Cov}_{t-2}\left[V_{t-1}, \mathrm{E}_{t-1}\left[r_{t}\right]\right]=0,
$$

was man im Allgemeinen nicht erwarten werden kann. In ökonomischen Anwendungen wird man Bedingung (9) wahrscheinlich in der Regel schärfer treffen und gleich deterministische erwartete Renditen $\left.\mathrm{E}_{t-1}\left[r_{t}\right]\right]$ unterstellen. 5

Werden die obigen Berechnungen auf weitere Perioden ausgedehnt, erhält man

$$
V_{t}=\sum_{\tau=t+1}^{T} \frac{\mathrm{E}_{t}\left[d_{\tau}\right]}{\prod_{j=1}^{\tau-t}\left(1+k_{t, t+j}\right)}
$$

mit den Kapitalkosten

$$
k_{t, t+j}=\mathrm{E}_{t}\left[r_{t+j}\right]+\frac{\operatorname{Cov}_{t}\left[V_{t+j-1}, \mathrm{E}_{t+j-1}\left[r_{t+j}\right]\right]}{\mathrm{E}_{t}\left[V_{t+j-1}\right]} .
$$

Dies ist die Standard-Lehrbuchformel zur Bewertung von unsicheren Investitionen mit zeitabhängigen Kapitalkosten. Zur Bestimmung eines Unternehmenswertes werden in diesem Rahmen Informationen über die erwarteten zukünftigen Zahlungsüberschüsse und die erwarteten zukünftigen einperiodigen Renditen sowie die Korrelation der einperiodigen Renditen mit den zukünftigen Unternehmenswerten benötigt. Da die zukünftigen Unternehmenswerte sich im wesentlichen aus den Renditen ergeben, können letztere als Autokorrelation der Renditen untereinander verstanden werden. Unter Verwendung der Kursgewinne $g_{t, t+j}$ von Periode $t$ bis Periode $t+1$, wobei

$$
V_{t+j}=V_{t}\left(1+g_{t, t+j}\right),
$$

kann der Quotient in (11) wie folgt geschrieben werden:

$$
\frac{\operatorname{Cov}_{t}\left[g_{t, t+j-1}, \mathrm{E}_{t+j-1}\left[r_{t+j}\right]\right]}{1+\mathrm{E}_{t}\left[g_{t, t+j-1}\right]}
$$

\footnotetext{
${ }^{5}$ So zum Beispiel Laitenberger und Löffler (2002).
} 


\subsection{Rendite und Zustandspreise}

Fügt man die Bewertungsgleichungen aus Abschnitt2.1 in die Definition der Rendite (8) ein, so erhält für den Unternehmenswert in $t$ die Gleichung:

$V_{t}=\mathrm{E}_{t}\left[\frac{\pi_{t+1}}{\pi_{t}}\left(V_{t+1}+d_{t+1}\right)\right]=\operatorname{Cov}_{t}\left[\frac{\pi_{t+1}}{\pi_{t}}, V_{t+1}+d_{t+1}\right]+\mathrm{E}_{t}\left[\frac{\pi_{t+1}}{\pi_{t}}\right] \mathrm{E}_{t}\left[V_{t+1}+d_{t+1}\right]$,

bzw. für die Rendite $r_{t+1}$ :

$$
1=\operatorname{Cov}_{t}\left[\frac{\pi_{t+1}}{\pi_{t}}, r_{t+1}\right]+\mathrm{E}_{t}\left[\frac{\pi_{t+1}}{\pi_{t}}\right] \mathrm{E}_{t}\left[1+r_{t+1}\right]
$$

Umgeformt und nach $\mathrm{E}_{t}\left[r_{t+1}\right]$ aufgelöst, erhält man unter Ausnutzung der Beziehung (3):

$$
1+\mathrm{E}_{t}\left[r_{t+1}\right]=\left(1-\operatorname{Cov}_{t}\left[\frac{\pi_{t+1}}{\pi_{t}}, r_{t+1}\right]\right)\left(1+i_{t+1}\right) .
$$

Wird der Quotient $\frac{\pi_{t+1}}{\pi_{t}}$ als Auszahlung eines Wertpapiers zum Zeitpunkt $t+1$ betrachtet, so kann auch für dieses Wertpapier eine Rendite $r_{\pi, t+1}$ berechnet werden:

$$
1+r_{\pi, t+1}=\frac{\frac{\pi_{t+1}}{\pi_{t}}}{\mathrm{E}_{t}\left[\frac{\pi_{t+1}}{\pi_{t}} \frac{\pi_{t+1}}{\pi_{t}}\right]} .
$$

Damit schreibt sich Gleichung 12 wie folgt:

$$
1+\mathrm{E}_{t}\left[r_{t+1}\right]=\left(1-\operatorname{Cov}_{t}\left[r_{\pi, t+1}, r_{t+1}\right] \mathrm{E}_{t}\left[\left(\frac{\pi_{t+1}}{\pi_{t}}\right)^{2}\right]\right)\left(1+i_{t+1}\right) .
$$

Unter Ausnutzung der Beziehung

$$
\operatorname{Var}_{t}\left[r_{\pi, t+1}\right]=\frac{\mathrm{E}_{t}\left[\left(\frac{\pi_{t+1}}{\pi_{t}}\right)^{2}\right]}{\mathrm{E}_{t}\left[\left(\frac{\pi_{t+1}}{\pi_{t}}\right)^{2}\right]^{2}}-\frac{\mathrm{E}_{t}\left[\frac{\pi_{t+1}}{\pi_{t}}\right]^{2}}{\mathrm{E}_{t}\left[\left(\frac{\pi_{t+1}}{\pi_{t}}\right)^{2}\right]^{2}},
$$

kann die letzte Gleichung schließlich zu der bekannten Kapitalmarktgleichung

$$
\mathrm{E}_{t}\left[r_{t+1}\right]=i_{t+1}+\frac{\mathrm{E}_{t}\left[r_{\pi, t+1}\right]-i_{t+1}}{\operatorname{Var}_{t}\left[r_{\pi, t+1}\right]} \operatorname{Cov}_{t}\left[r_{t+1}, r_{\pi, t+1}\right]
$$

umgeformt werden. Die letzte Gleichung drückt die erwartete Rendite der Investition als Regressionsgleichung der Rendite mit der Rendite des Wertpapiers $\frac{\pi_{t+1}}{\pi_{t}}$ aus. $r_{\pi, t+1}$ nimmt in diesem Rahmen die Rolle des Indexes ein, die zum Beispiel beim CAPM durch die Rendite des Marktportfolios eingenommen wird. 
Wie bei Fama (1977) können nun Bedingungen an die einzelnen Bestandteile der Kapitalkostenformel (13) hergeleitet werden, die eine Autokorrelation der Renditen verhindern und damit eine bedenkenlose Verwendung der erwarteten Renditen als Kapitalkosten ermöglichen. Ersetzt man bei Fama konsequent das Marktportfolio durch das Wertpapier $\frac{\pi_{t+1}}{\pi_{t}}$, dann garantieren ein sicherer kurzfristiger Zinssatz $i_{t+1}$, ein sicherer Preis des Risikos des Bewertungsportfolios $\frac{\mathrm{E}_{t}\left[r_{\pi, t+1}\right]-i_{t+1}}{\operatorname{Var}_{t}\left[r_{\pi, t+1}\right]}$ sowie eine Korrelation $\operatorname{Cov}_{t}\left[r_{t+1}, r_{\pi, t+1}\right]$, die zu allen früheren Zeitpunkten $\tau<t$ bereits mit Sicherheit bekannt ist, eine sichere erwartete Rendite $\mathrm{E}_{t}\left[r_{t+1}\right]$, wobei 'sicher' in diesem Kontext immer bedeutet, dass die entsprechenden $\mathcal{F}_{t}$-messbaren Zufallsvariablen sogar $\mathcal{F}_{0}$-messbar sind, also zum Beispiel $\mathrm{E}_{t}\left[r_{t+1}\right]=\mathrm{E}\left[r_{t+1}\right]$.

\section{Risikoauflösung und Kapitalkosten}

Im verbleibenden Teil dieses Aufsatzes sollen für bestimmte Beispiele von Dividenden- und Zustandspreisprozesse die sich ergebenden Kapitalkosten untersucht werden und insbesondere auf ihre Autokorrelation getestet werden. In diesem Rahmen wurde in den letzten Jahren häufig von der Auflösung der Unsicherheit gesprochen, wobei insbesondere die Fälle einer schlagartigen und einer gleichmäßigen Auflösung behandelt wurden ${ }^{6}$ Grundsätzlich kann unter der Auflösung der Unsicherheit die Entwicklung der Filtration $\left(\mathcal{F}_{t}\right)_{t=0, \ldots, T}$ verstanden werden. Je feiner die Partition $\mathcal{F}_{t}$ den Zustandsraum $\Omega$ abdeckt, desto genauer ist das Wissen der Marktteilnehmer bezüglich der möglichen zukünftigen Entwicklungen. Für die Wertfindung von Relevanz sind allerdings nur solche Veränderungen, die einen Einfluss auf die Verteilung der zukünftigen Dividenden als auch auf die Verteilung der Zustandspreise haben. Von einer Veränderung von Periode $t \mathrm{zu} t+1$ einer Verteilung einer Zufallsvariable $x_{\tau}$ mit $\tau \geq t+1$ kann gesprochen werden, wenn die bedingte Verteilung der Zufallsvariable $x_{\tau} F_{x}\left(s \mid \mathcal{F}_{t+1}\right)$, gegeben die Entwicklung bis zum Zeitpunkt $t+1$, sich von der bedingten Verteilung $F_{x}\left(s \mid \mathcal{F}_{t}\right)$, gegeben die Entwicklung bis $t$, unterscheidet.

Eine schlagartige Auflösung des Risikos im Zeitpunkt $\tau$ einer Zufallsvariable $x_{\tau}$ ist dann gegeben, wenn die bedingten Verteilungen $F_{x}\left(s \mid \mathcal{F}_{t}\right)$ bis zum Zeitpunkt $t<\tau$ sich nicht von der absoluten Verteilung $F_{x}(s)=$ $F_{x}\left(s \mid \mathcal{F}_{0}\right)$ unterscheiden. Damit wird zum Ausdruck gebracht, dass bis zum Zeitpunkt $\tau-1$ nichts über die Verteilung von $x_{\tau}$ dazugelernt wird. Erst in Periode $\tau$ wird die gesamte Unsicherheit aufgelöst.

\footnotetext{
${ }^{6}$ Ballwieser (1993), Schwetzler (2000), Wilhelm (2003).
} 
Schwieriger ist die Abgrenzung dessen, was unter einer gleichmäßigen Auflösung des Risikos zu verstehen ist. Man betrachte dazu die Zufallsvariablen

$$
\rho_{t+1}:=\frac{\mathrm{E}_{t+1}\left[x_{\tau}\right]}{\mathrm{E}_{t}\left[x_{\tau}\right]}-1
$$

Diese sind per Definition $\mathcal{F}_{t+1}$-messbar. Es gilt offensichtlich:

$$
d_{T}=\mathrm{E}\left[d_{T}\right]\left(1+\rho_{1}\right)\left(1+\rho_{2}\right) \cdots\left(1+\rho_{T}\right) .
$$

Wilhelm (2003) schlägt vor, von gleichmäßiger Auflösung des Risikos zu sprechen, wenn die Varianz aller $\rho_{t}$ gleich ist. In diesem Aufsatz wird die gleichmäßige Auflösung der Unsicherheit noch etwas strenger gefasst, indem gefordert wird, dass alle $\rho_{t}$ unabhängig und identisch verteilt sein sollen. Offensichtlich weist eine Zufallsvariable, die nach diesem Kriterium eine gleichmäßige Auflösung der Unsicherheit aufweist, dies auch nach dem Wilhelmschen Kriterium. Umgekehrt gilt dies nicht, da Verteilungen mit gleicher Varianz vollkommen unterschiedliche Formen aufweisen können 7

Im Folgenden soll untersucht werden, welche Auswirkungen auf die Kapitalkostenformeln unterschiedliche Szenarien der Risikoauflösung sowohl der Dividenden als auch der Zustandspreise haben können. Dabei werden nur Investitionen mit einmaligen Zahlungen betrachtet. Der Fall mehrmaliger Zahlungen bringt keine zusätzlichen Erkenntnisse mit sich. Die Analyse würde dadurch nur erschwert, indem die Zahlungen bzw. deren Erwartungswerte in früheren Perioden miteinander korrelieren können 8

Als erstes wird der Fall einer schlagartigen Auflösung der Unsicherheit untersucht. Man betrachte den Extremfall einer sicheren Zahlung $d_{T}$ in Periode T. Da keine Unsicherheit im Spiel ist, kann hier sowohl von einer schlagartigen als auch einer gleichmäßigen Auflösung gesprochen werden. Über $T$ Perioden hat $d_{T}$ eine sichere Rendite von

$$
r_{0, T}=\frac{d_{T}}{\mathrm{E}\left[\pi_{T} d_{T}\right]}-1=i_{0, T} .
$$

Über eine einzelne Periode ergibt sich jedoch die Rendite

$$
1+r_{t+1}=\frac{V_{t+1}}{V_{t}}=\frac{\mathrm{E}_{t+1}\left[\pi_{T} d_{T}\right]}{\pi_{t+1}} \frac{\pi_{t}}{\mathrm{E}_{t}\left[\pi_{T} d_{T}\right]}=\frac{1+i_{t, T}}{1+i_{t+1, T}},
$$

\footnotetext{
${ }^{7}$ Als ein Beispiel seien die Standardnormalverteilung und die diskrete Verteilung, bei der mit gleicher Wahrscheinlichkeit die Werte -1 und 1 angenommen werden, genannt. Beide Verteilungen haben eine Varianz von eins.

${ }^{8}$ Wer bei den folgenden Ausführungen trotzdem unbedingt mehrmalige Zahlungen unterstellen will, kann sich vorstellen, es gelte für alle $d_{t}$, dass $d_{t}=\alpha_{t} V_{t}$ mit einem skalaren $\alpha_{t} \in \mathbb{R}$.
} 
die in $t$ einen Erwartungswert von

$$
\mathrm{E}_{t}\left[\frac{1+i_{t, T}}{1+i_{t+1, T}}\right]=1+i_{t, t+1}
$$

aufweist. Zum Zeitpunkt $t=0$, in dem die Bewertung durchgeführt wird, ist dies eine unsichere Rendite, wenn die kurzfristigen Zinssätze $i_{t, t+1}$ nicht deterministisch sind. Dies zeigt, dass selbst der allertrivialste Fall einer einzelnen sicheren Zahlung sich nicht ohne weiteres mit ihren erwarteten einperiodigen Renditen diskontieren lässt. Nur wenn die kurzfristigen Zinssätze untereinander unkorreliert sind, ist eine Anwendung des Kapitalkostenkalküls möglich.

Im Allgemeinen gilt für die Rendite von Periode $t$ nach Periode $t+1$ einer unsicheren Zahlung $d_{T}$ in Periode $T$ :

$$
1+r_{t+1}=\frac{\mathrm{E}_{t+1}\left[\frac{\pi_{T}}{\pi_{t+1}} d_{T}\right]}{\mathrm{E}_{t}\left[\frac{\pi_{T}}{\pi_{t}} d_{T}\right]} .
$$

Mit der Schreibweise für die Auflösung der Unsicherheit aus Gleichung (14) kann man die Erwartungswerte wie folgt schreiben:

$$
\mathrm{E}_{t}\left[\frac{\pi_{T}}{\pi_{t}} d_{T}\right]=\mathrm{E}\left[d_{T}\right]\left(1+\rho_{1}\right) \cdots\left(1+\rho_{t}\right) \mathrm{E}_{t}\left[\frac{\pi_{T}}{\pi_{t}}\left(1+\rho_{t+1}\right) \cdots\left(1+\rho_{T}\right)\right] .
$$

Durch Erweiterung mit $\frac{\pi_{t+1}}{\pi_{t+2}}$, usw... erhält man dadurch für die Rendite den folgenden Ausdruck:

$$
1+r_{t+1}=\left(1+\rho_{t+1}\right) \frac{\mathrm{E}_{t+1}\left[\left(\frac{\pi_{t+2}}{\pi_{t+1}}\left(1+\rho_{t+2}\right)\right) \cdots\left(\frac{\pi_{T}}{\pi_{T-1}}\left(1+\rho_{T}\right)\right)\right]}{\mathrm{E}_{t}\left[\left(\frac{\pi_{t+1}}{\pi_{t}}\left(1+\rho_{t+1}\right)\right) \cdots\left(\frac{\pi_{T}}{\pi_{T-1}}\left(1+\rho_{T}\right)\right)\right]} .
$$

Unter der Voraussetzung, dass $\mathrm{E}_{t}\left[\frac{\pi_{t+1}}{\pi_{t}}\left(1+\rho_{t+1}\right)\right]$ für alle $t$ deterministisch ist 9 kann dies so aufgelöst werden, dass

$$
1+r_{t+1}=\left(1+\rho_{t+1}\right) \frac{1}{\mathrm{E}_{t}\left[\frac{\pi_{t+1}}{\pi_{t}}\left(1+\rho_{t+1}\right)\right]} .
$$

Der Diskontierungsfaktor $\frac{1}{1+k_{t+1}}$ ergibt sich als

$$
\frac{1}{1+k_{t+1}}=\frac{1}{1+\mathrm{E}_{t}\left[r_{t+1}\right]}=\mathrm{E}_{t}\left[\frac{\pi_{t+1}}{\pi_{t}}\left(1+\rho_{t+1}\right)\right]
$$

\footnotetext{
${ }^{9}$ Streng genommen genügt sogar die Bedingung, dass die $\mathrm{E}_{t}\left[\frac{\pi_{t+1}}{\pi_{t}}\left(1+\rho_{t+1}\right)\right]$ paarweise unabhängig sind.
} 
Damit wurde gezeigt, dass unter den Voraussetzungen, die eine Anwendung des Kapitalkostenkalküls erst möglich machen, die Stochastik der einperiodigen Renditen einer Investition identisch ist mit der Stochastik, mit der sich die Unsicherheit in der gleichen Periode auflöst. Die Wahrscheinlichkeitsverteilungen der beiden Zufallsvariablen $\left(1+r_{t}\right)$ und $\left(1+\rho_{t}\right)$ unterscheiden sich nur um einen skalaren Faktor, der wiederum von der Auflösung der Unsicherheit und dem Bewertungsfaktor der besagten Periode abhängt. Wenn die Kapitalkosten gleich der erwarteten Rendite sind, so ergeben sie sich als der Kehrwert des bewerteten Informationszuflusses.

Um der Bedingung

$$
\mathrm{E}_{t}\left[\frac{\pi_{t+1}}{\pi_{t}}\left(1+\rho_{t+1}\right)\right] \quad \text { deterministisch }
$$

einen ökonomischen Sinn zu geben, setzt man wie in Abschnitt 2.3

$$
\frac{\pi_{t+1}}{\pi_{t}}=\frac{1+r_{\pi, t+1}}{1+i_{t+1}}\left(\frac{i_{t+1}-\mathrm{E}_{t}\left[r_{\pi, t+1}\right]}{\operatorname{Var}_{t}\left[r_{\pi, t+1}\right]}\right)
$$

ein, mit der Rendite $r_{\pi, t+1}$ des Bewertungsindexes von $t$ nach $t+1$ :

$\mathrm{E}_{t}\left[\frac{\pi_{t+1}}{\pi_{t}}\left(1+\rho_{t+1}\right)\right]=\frac{1}{1+i_{t+1}}\left(\frac{i_{t+1}-\mathrm{E}_{t}\left[r_{\pi, t+1}\right]}{\operatorname{Var}_{t}\left[r_{\pi, t+1}\right]}\right) \mathrm{E}_{t}\left[\left(1+r_{\pi, t+1}\right)\left(1+\rho_{t+1}\right)\right]$.

Damit wird deutlich, dass Annahmen an die Auflösung der Unsicherheit für die zu bewertende Zahlung alleine nicht ausreichen, um eine Bewertung mit dem Kapitalkostenkonzept unter Verwendung der erwarteten Renditen zu gewährleisten. Nur wenn auch die kurzfristigen Zinsen, die erwarteten Renditen der Bewertungsportfolios, deren Varianz und die Kovarianz 10 mit dem Informationsprozess $\rho_{t}$ von Anbeginn bekannte Größen sind, erhält man die gewünschten Ergebnisse.

Als Nächstes soll das bisher Erreichte auf den Fall einer unsicheren Zahlung $d_{T}$ in $T$, deren Unsicherheit sich schlagartig auflöst, angewendet werden. Das bedeutet, dass in Perioden $t<T$ keine Auflösung der Unsicherheit stattfindet, oder anders ausgedrückt: $\rho_{t}=0$. Die Bedingung (17) impliziert nun, dass $\mathrm{E}_{t}\left[\frac{\pi_{t+1}}{\pi_{t}}\right]$ deterministisch ist, was gleichbedeutend ist mit deterministischen kurzfristigen Zinsen. In einer Periode $t<T$ erhält man mit

${ }^{10}$ Die Kovarianz taucht hier wieder wegen der Kovarianzidentität auf:

$$
\mathrm{E}_{T-1}\left[\left(1+r_{\pi, T}\right) d_{T}\right]=\operatorname{Cov}_{T-1}\left[r_{\pi, T}, d_{T}\right]+\mathrm{E}_{T-1}\left[1+r_{\pi, T}\right] \mathrm{E}_{T-1}\left[d_{T}\right] .
$$


Gleichung (16):

$$
1+r_{t}=\frac{1}{\mathrm{E}_{t-1}\left[\frac{\pi_{t}}{\pi_{t-1}}\right]}=1+i_{t}
$$

Der Fall einer gleichmäßigen Auflösung von Unsicherheit lässt sich mit Gleichung (16) ebenso elegant untersuchen. Da $\mathrm{E}_{t}\left[\frac{\pi_{t+1}}{\pi_{t}}\left(1+\rho_{t+1}\right)\right]$ neben der Stochastik des Informationsprozesses vom risikolosen Zins und von der Rendite des Bewertungsportfolios abhängt, wird man auch bei einer gleichmäßigen Auflösung von Unsicherheit konstante Kapitalkosten nur dann erhalten, wenn auch der risikolose Zins, der Marktpreis des Risikos und die Kovarianz der Rendite des Bewertungsportfolios mit dem Informationsprozess konstante Größen sind. Mithin wird man dann in der Regel davon ausgehen können, dass auch die Renditen des Bewertungsindexes identisch und unabhängig verteilt sind.

\section{Schluss}

In dieser Arbeit wurde gezeigt, dass die Bewertung von Investitionen auf arbitragefreien Märkten konsistent mit dem Kapitalkostenkalkül durchgeführt werden kann, sofern die Kapitalkosten adäquat ermittelt werden. Die in der Praxis als Kapitalkosten in der Regel verwendeten erwarteten Renditen sind allerdings nur dann geeignet, wenn die einperiodigen Renditen unkorreliert sind. Ist dies nicht der Fall, so muss zu den erwarteten Renditen ein Korrekturterm hinzugefügt werden.

Für den Fall unkorrelierter Renditen kann in dieser Arbeit ein enger Zusammenhang der einperiodigen Renditen mit dem Prozess der Auflösung von Unsicherheit festgestellt werden. Dies steht im Gegensatz zu Ergebnissen von Wilhelm (2003), der für eine andere Definition der Unsicherheitsauflösung keine Zusammenhänge zwischen Informationsfluss und Kapitalkosten feststellen kann. Das liegt zum einen daran, dass Wilhelm additive Zuwächse der Information betrachtet, zum anderen an einer etwas ungeschickten Definition des Kapitalkostenkonzeptes bei Wilhelm. Mit der in dieser Arbeit verwendeten Definition der Kapitalkosten und unter Bedingungen, die es erlauben die erwarteten Renditen der Investition als Kapitalkosten heranzuziehen, ergeben sich sehr einfache und intuitive Zusammenhänge zwischen dem Informationsprozess und den Renditen bzw. den Kapitalkosten. 


\section{Literatur}

Ang, A. und Liu, J.: 2002, How to discount cashflows with time-varying expected returns. Discussion Paper, Columbia University.

Ballwieser, W.: 1993, Methoden der Unternehmensbewertung, in G. Gebhardt, W. Gerke and M. Steiner (Hrsg.), Handbuch des Finanzmanagements, 151-176.

Bamberg, G. und Baur, F.: 2001, Statistik, 11. Aufl., Oldenbourg, München, Wien.

Brennan, M.: 1997, The term structure of discount rates, Financial Management 26, 81-90.

Fama, E. und French, K.: 1988, Permanent and temporary components of stock prices, Journal of Political Economy 96 246-273.

Fama, E.: 1977, Risk-adjusted discount rates and capital budgeting under uncertainty, Journal of Financial Economics 5, 3-24.

Laitenberger, J. und Löffler, A.: 2002, Capital budgeting in arbitrage-free markets. Discussion Paper, Universität Hannover.

Lo, A. und Mackinlay, A.: 1988, Stock market prices do not follow random walks: evidence from a simple specification test, Review of Financial Studies, xx 41-66.

Poterba, J. und Summers, L.: 1988, Mean reversion in stock prices, Journal of Financial Economics 22, 27-59.

Rapp, M.-S.: 2003, Arbitragefreie Bewertung von Investitionsprojekten Ein Brückenschlag zwischen No-Arbitrage-Theorie und DCF-Verfahren mittels stochastischer Diskontierungssätze. Diskussionspapier Handelshochschule Leipzig.

Schwetzler, B.: 2000, Unternehmensbewertung unter Unsicherheit - Sicherheitsäquivalent- oder Risikozuschlagsmethode?, Zeitschrift für betriebswirtschaftliche Forschung 52, 469-486.

Wilhelm, J.: 2003, Unternehmensbewertung - eine finanzmarkttheoretische Untersuchung. Passauer Diskussionspapiere.

Wilhelm, J.: 1985, Arbitrage Theory, Springer, Berlin et al. 